\title{
Carcinoma de células claras de ovario, a propósito de
}

\section{un caso}

\section{Clear cell carcinoma of the ovary, about a case}

1 Ihosvannys Enrique Carreño Rolando (iD) http://orcid.org/0000-0003-1259-3491 Hospital Universitario Comandante Faustino Pérez Hernández, Matanzas ihosvanny.mtz@infomed.sld.cu

2 Mildrey García Hernández (iD) http://orcid.org/0000-0002-7247-1384 Hospital Universitario Comandante Faustino Pérez Hernández, Matanzas mildreyg.mtz@infomed.sld.cu

3 Luís Enrique Curbelo Gutiérrez $\quad$ (iD http://orcid.org/0000-0002-7761-9595 Hospital Universitario Comandante Faustino Pérez Hernández, Matanzas luis.curbelo@icloud.com

4 Giannina de los Dolores Arroba Rios $\quad$ (iD) http://orcid.org/0000-0002-8701-6296 Hospital Santa Inés Ambato, Ecuador garrobar@hotmail.com

Artículo de Investigación Científica y Tecnológica Enviado: 07/11/2021

Revisado: 22/11/2021

Aceptado: 03/12/2021

Publicado:05/01/2022

DOI: https://doi.org/10.33262/anatomiadigital.v5i1.1967

G. de los D. (2022). Carcinoma de células claras de ovario, a propósito de un caso.

Cítese: $\quad$ Anatomía Digital, 5(1), 26-36. https://doi.org/10.33262/anatomiadigital.v5i1.1967

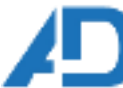

Ciencia Digital

ANATOMÍA DIGITAL, es una Revista Electónica, Trimestral, que se publicará en soporte electrónico tiene como misión contribuir a la formación de profesionales competentes con visión humanística y crítica que sean capaces de exponer sus resultados investigativos y científicos en la misma medida que se promueva mediante su intervención cambios positivos en la sociedad. https://anatomiadigital.org

La revista es editada por la Editorial Ciencia Digital (Editorial de prestigio registrada en la Cámara Ecuatoriana de Libro con No de Afiliación 663) www.celibro.org.ec 
Palabras

claves:

tumores de

ovario,

carcinoma de

células claras, mutación

ARID1A.

\section{Keywords:}

ovarian tumors, clear cell carcinoma,

ARID1A

mutation.

\section{Resumen}

Introducción: La clasificación histológica de los tumores ováricos por la Organización Mundial de la Salud (OMS) se basa en principios histogenéticos, y esta divide los tumores ováricos de acuerdo a su derivación de células epiteliales, células germinales y mesenquimales (el estroma y el cordón sexual). Objetivo: describir las características clínico- epidemiológicas del carcinoma de células claras de ovario. Metodología: hicimos una revisión de la bibliografía sobre el tema y presentamos los resultados. Resultados: Se ha notificado que el carcinoma de células claras (CCC) del ovario tiene un pronóstico peor que el resto de las neoplasias de este grupo. Teniendo en cuenta esto, se ha llevado a cabo esta presentación de un caso atendido en nuestra institución, con un tratamiento efectivo, válido por la sobrevida de la paciente y una buena calidad de vida. Conclusiones: se trata de un tumor de ovario epitelial frecuentemente resistente a la quimioterapia convencional

\section{Abstract}

Introduction: The histological classification of surgery tumors by the World Health Organization (WHO) is based on histogenetic principles, and it divides surgery tumors according to their derivation of epithelial cells, germ cells and mesenchymal cells (stroma and sex cord). Objective: to describe the clinicalepidemiological characteristics of clear cell ovarian carcinoma. Methodology: we made a review of the bibliography on the subject and presented the results. Results: Clear cell carcinoma (CCC) of the ovary has been reported to have a worse prognosis than all other neoplasms in this group. Taking this into account, this presentation of a case attended in our institution has been carried out, with an effective treatment, valid for the survival of the patient and a good quality of life. Conclusions: it is an epithelial ovarian tumor frequently resistant to conventional chemotherapy. 


\section{Introducción}

La clasificación histológica de los tumores ováricos por la Organización Mundial de la Salud (OMS) se basa en principios histogenéticos, y esta divide los tumores ováricos de acuerdo a su derivación de células epiteliales, células germinales y mesenquimales (el estroma y el cordón sexual). Los tumores ováricos epiteliales, que son la mayoría de los tumores ováricos malignos, se agrupan además en tipos histológicos de la siguiente manera: seroso, mucinoso, endometrioides, células claras, tumores de células de transición (tumores de Brenner), carcinosarcoma, tumor epitelial mixto, carcinoma indiferenciado y otros. ${ }^{(1)}$

El carcinoma epitelial ovárico (CEO) es una enfermedad heterogénea con subtipos histológicos que muestran características citogenéticas, firmas moleculares, vías de señalización oncogénica y comportamiento clínico-biológico diferentes. Los recientes avances en histopatología y citogenética han proporcionado información sobre las características fisiopatológicas y la historia natural de este grupo. Varios estudios han demostrado que los carcinomas serosos, endometrioides y de células claras de alto o bajo grado se caracterizan por mutaciones que involucran los genes TP53, K-ras/BRAF, CTNNB1 y PIK3CA, respectivamente. Los carcinomas serosos de alto grado, el subtipo más común, a menudo se manifiestan con la propagación trancelómica temprana de la enfermedad más allá de los ovarios. Sobre la base de mecanismos patógenos, los hallazgos recientes sugieren un modelo dualista de carcinogénesis ovárica que consiste en los tipos I y II. Los cánceres de tipo I (serosos, mucinosos y endometrioides de bajo grado) comúnmente surgen de lesiones precursoras bien descritas y genéticamente estables; se manifiestan como grandes masas anexiales con enfermedad en estadio temprano; y tienen un curso clínico relativamente indolente, con un buen pronóstico general. Por el contrario, los carcinomas de tipo II (variantes serosas, endometrioides, mixtas e indiferenciadas de alto grado) a menudo demuestran inestabilidad cromosómica y tienen un comportamiento biológico agresivo. Un mejor conocimiento de los síndromes hereditarios del cáncer de ovario y las anomalías citogenéticas asociadas ha llevado a un mayor interés en nuevos biomarcadores y terapias moleculares. ${ }^{(2)}$

El CEO es la principal causa de muerte en mujeres con neoplasias malignas ginecológicas. Entre sus diferentes subtipos el carcinoma de células claras (CCC) difiere de los otros tipos histológicos con respecto a sus características clínicas. Los cambios moleculares en el CCC siguen siendo en gran medida desconocidos. En el contexto de la biología del CCC se han analizado varios estudios recientes sobre la pérdida de la heterocigosidad $(\mathrm{LOH})$, la pérdida alélica, la hibridación genómica comparativa, la mutación, el estado de metilación, el perfil de expresión génica de microarrays y la proteómica. El daño del ADN causado por el estrés oxidativo es un factor crítico en el 
proceso cancerígeno. Los estudios han implicado la participación de regiones cromosómicas específicas (5q, 6q, 9p, 10q, 11q, 17q y 22q). Además, los genes PTEN y APC (evento temprano), p53, quinasas similares a polos, Emi1 y K-RAS (evento tardío) pueden estar involucrados en su carcinogénesis. ${ }^{(3)}$

Diferentes tipos histológicos de cáncer de ovario epitelial pueden representar diferentes enfermedades con características clínicas y moleculares únicas. Se ha notificado que el carcinoma de células claras (CCC) del ovario tiene un pronóstico peor que el cáncer de ovario epitelial seroso de alto grado. En un artículo publicado por del Carmen MG, et all. se revisa críticamente la literatura pertinente a la patología, patogénesis, diagnóstico, manejo y resultado de pacientes con CCC ovárico. Como resultado de este estudio podemos plantear que los tumores de CCC ováricos representan entre el 5 y el $25 \%$ de los cánceres de ovario. Su diagnóstico histológico puede ser difícil, resultando a menudo en la clasificación errónea de estos tumores. El CCC ovárico tiende a presentarse en etapas tempranas y se ha asociado con mutaciones de endometriosis, ARID1A y PIK3CA. En comparación con los controles de estadio, los pacientes con CCC de ovario en estadio temprano pueden tener un mejor pronóstico que los pacientes con tumores serosos de alto grado. Para aquellos con enfermedad en estadio avanzado, la histología serosa de alto grado confiere un mejor pronóstico que el CCC ovárico. Finalmente concluyen el artículo expresando que el CCC ovárico es una entidad biológicamente distinta y los estudios futuros deben explorar el papel de las terapias dirigidas en su manejo. ${ }^{(4)}$

El contenido de quistes endometrióticos y altas concentraciones de hierro libre, son posibles causas de la carcinogénesis a través del estrés oxidativo persistente inducido por hierro. Varios estudios indican que el microambiente tumoral induce perfiles específicos de expresión génica que contribuyen al desarrollo de subtipos de cáncer distintos. ${ }^{(5)}$

La mejora de la especificidad de la definición histológica de este tipo de tumor está ayudando a estos esfuerzos, pero, debido a la rareza del carcinoma de células claras, la colaboración internacional será esencial para diseñar ensayos clínicos adecuados y a gran escala, teniendo esto en cuenta, decidimos presentar un caso atendido en nuestra institución, con el objetivo de describir las características clínicas y las principales opciones de manejo en este tipo de tumores.

\section{Metodología}

Varias bases de datos de PubMed y la Biblioteca Nacional de Medicina fueron buscadas de 2015 a 2020 para estudios en todo el mundo que fueron publicados en inglés y traducidas al español. Después de examinar todos los resúmenes de las publicaciones identificadas por la búsqueda inicial, se incluyeron en el análisis estudios e informes de 
casos sobre mujeres con CCC de ovario. La idoneidad de los estudios se definió a los efectos de esta revisión como la notificación de las características clínicas o biológicas, el tratamiento o los resultados clínicos de los pacientes con este diagnóstico.

\section{Resultados}

Se trata de una paciente de 81 años de edad, con antecedentes de Diabetes Mellitus tipo 2 que se controla solamente con tratamiento dietético, con fórmula ginecológica Gestaciones 10, Partos 10, Abortos 0, Número de parejas sexuales: 1, Menarquia a los 11 años, tuvo su primera relación sexual a los 17 años, su primer embarazo a los 17 años y Menopausia a los 50 años. Refiere que hace tres meses comenzó con dolor abdominal bajo lateralizado a la izquierda por lo que acudió a consulta.

\section{Examen Físico}

Abdomen: suave, depresible, no doloroso a la palpación, no visceromegalia y ruidos hidroaéreos presentes y normales, con maniobras de Tarral y Moneda de Pitres positivas.

Tacto vaginal: anejo izquierdo irregular a la palpación, con aumento de la consistencia, poco doloroso al tacto y de tamaño normal

Examen con espéculo: negativo

Tacto rectal: negativo

Teniendo en cuenta los hallazgos al examen físico, se realizó Ultrasonido que en abdomen informó la presencia de líquido libre en cavidad abdominal y ginecológico que informa útero de ecoestructura heterogénea con imagen ocupativa de espacio en anejo izquierdo (aproximadamente $5 \mathrm{~cm}$ ) y TAC pélvico abdominal que describió la misma imagen sin alteraciones a otros niveles. Con estos datos al examen físico y complementario se llevó a cabo la cirugía ginecológica, con biopsia de la lesión que informó: Carcinoma de células claras de ovario con marcada desmoplasia tumoral, diagnosticándose cáncer de ovario.

La paciente acudió a consulta central de ginecología donde se estadia etapa IIIC y se decidió tratamiento oncoespecífico con quimioterapia convencional esquema Carboplatino (5 AUC) + Paclitaxel $\left(175 \mathrm{mg} /{ }^{2}\right)$ día 1 en perfusión intravenosa de 3 horas en frecuencias de 6 ciclos cada 21 días sin obtener la respuesta adecuada, por lo que se decidió tratamiento de segunda línea con Gemcitabina $(1.250 \mathrm{mg} / \mathrm{m} 2)$ en perfusión intravenosa de 30 minutos, los días primero y octavo cada 21 días durante 6 ciclos, obteniéndose una adecuada respuesta tumoral. La paciente se mantiene en seguimiento y presenta buena calidad de vida. 


\section{Discusión}

Clínicamente, se ha sugerido que los CCC se desarrollan a partir de la endometriosis, pero ha habido poca evidencia molecular que respalde esta especulación. El análisis de microarrays reveló recientemente que el factor nuclear de hepatocito-1beta (HNF-1beta) fue significativamente alto en el carcinoma de células claras del ovario. En estudio realizado por Kato, et all. se examinaron 30 tumores de células claras (26 malignos, tres límites y uno benignos) y 40 quistes endometrióticos para aclarar si la diferenciación en el linaje celular claro ya comienza en la endometriosis ovárica. Todos los 30 tumores de células claras, incluidos los límites y benignos, mostraron expresión inmunohistoquímica de HNF-1beta en el núcleo, mientras que otros tipos de tumores epiteliales ováricos (endometrioides, serosos, mucinosos y tumores de Brenner) rara vez lo expresaron. La expresión de HNF-1beta se observó en endometriosis atípica (cuatro casos), o en endometriosis de naturaleza reactiva (cinco casos). Estos resultados indican que el HNF-1beta es un excelente marcador molecular para tumores de células claras ováricas, incluyendo lesiones benignas, límites y malignas. ${ }^{(6)}$

Existen diferencias geográficas y raciales significativas en la incidencia de carcinoma de células claras en comparación con otros tumores ováricos epiteliales. Los pacientes con carcinoma de células claras son más jóvenes, tienden a presentarse en una etapa temprana, y sus tumores se asocian comúnmente con la endometriosis, que es ampliamente aceptada como un precursor directo del carcinoma de células claras y se ha identificado patológicamente en aproximadamente el 50\% de los casos de carcinoma de células claras. Las alteraciones genéticas específicas más frecuentes e importantes en el carcinoma de células claras son las mutaciones del dominio de interacción rico en AT 1A (ARID1A) (50\% de los casos) y fosfatidilinositol-4,5-bisfosfato 3-quinasa subunidad catalítica alfa (PIK3CA). En términos más generales, se han identificado subgrupos de carcinoma de células claras basados en C-APOBEC (enzima de edición de ARNm B de apolipoproteína B, polipéptido catalítico similar) y firmas mutacionales CAGE (relacionadas con la edad. Los pacientes con enfermedad en estadio temprano tienen un resultado clínico favorable, pero el pronóstico de los pacientes con enfermedades de estadio avanzado o recurrente es pobre. Se requieren estrategias de tratamiento alternativo para mejorar el resultado del paciente y el desarrollo de terapias dirigidas basadas en características moleculares es un enfoque prometedor. ${ }^{(7)}$

En un estudio llevado a cabo por Lee Y- Y, et all. con el objetivo de comparar el resultado de supervivencia entre el carcinoma de células claras (CCC) y otros subtipos histológicos de carcinoma ovárico epitelial, realizado en el período de enero de 1974 a febrero de 2011, se identificaron un total de 31.800 pacientes (CCC; 2152, no CCC; 29648) de 12 estudios que cumplían los criterios de inclusión. Como conclusión este análisis sugiere que los pacientes con CCC ovárico tenían un pronóstico más pobre que 
aquellos con otros subtipos histológicos, especialmente en etapas avanzadas. Además, son necesarias diferentes estrategias de tratamiento para los pacientes con CCC ovárico. $^{(8)}$

Otro estudio llevado a cabo por $\mathrm{Ku}$, et all. con el objetivo de comparar los resultados clínicos de los pacientes taiwaneses con carcinomas de células claras ováricas (CCCC) y carcinomas serosos (CC) se llegó a la conclusión de que los resultados clínicos del CCC son generalmente más pobres. ${ }^{(9)}$

No se ha implementado una selección terapéutica clara basada en el perfil molecular para esta enfermedad. La mutación oncogénica de PIK3CA, que activa la vía de señalización PIK3CA/AKT/mTOR, es una alteración farmacológica prometedora. Estudios recientes han identificado la mutación ARID1A como otra alteración vinculada a la selección terapéutica basada en la letalidad sintética: mutaciones ariD1A hacen que las células claras sean sensibles a los fármacos dirigidos a polimerasa (ADP-ribosa) y EZH2, así como a inhibidores de la glutatión. Además, recientemente obtuvimos evidencia de que el CCC deficiente de ARID1A podría beneficiarse del tratamiento con gemcitabina. La medicina de precisión basada en el perfilado por alteración genética podría mejorar el pronóstico de estos pacientes. ${ }^{(10)}$

En este sentido podemos plantear que CCC de ovario es a menudo resistente a la quimioterapia convencional y estándar mediante medicamentos citotóxicos. Sin embargo, alberga una característica genómica única de frecuencia aproximada de un 50\% que es la deficiencia de ARID1A. Se llevó a cabo un estudio por Kuroda, et all. para investigar opciones quimioterapéuticas estándar adecuadas para pacientes con carcinoma de células pequeñas de ovario con deficiencia de ARID1A. Para ello se identificaron fármacos con toxicidad selectiva para células CCC deficientes por ARID1A entre seis fármacos citotóxicos utilizados en quimioterapia estándar para CCC. Los efectos antitumorales del tratamiento farmacológico se evaluaron utilizando un modelo de xenoinjerto. Se analizaron muestras y casos de pacientes para la asociación entre la respuesta terapéutica y la deficiencia de ARID1A. Como resultados las células CCC con deficiencia de ARID1A tenían sensibilidad selectiva a la gemcitabina. Los valores de IC50 para gemcitabina de células con deficiencia de ARID1A fueron significativamente más bajos que los de las células con dominio de ARID1A (p a 0.0001). El crecimiento de xenoinjertos OCCC con deficiencia de ARID1A se inhibió mediante la administración de gemcitabina, y el tratamiento con gemcitabina indujo efectivamente apoptosis en células CCC deficientes en ARID1A. Un caso deficiente de ARID1A que era resistente a múltiples fármacos citotóxicos, incluyendo paclitaxel más carboplatino en el adyuvante y etopósido más irinotecán en el tratamiento de primera línea, exhibió una respuesta dramática a gemcitabina en el tratamiento de segunda línea. 
Como principal conclusión: Los pacientes con CCC con deficiencia de ARID1A podrían beneficiarse del tratamiento con gemcitabina en entornos clínicos. ${ }^{(11)}$

A pesar de estos avances, se necesitan nuevos enfoques específicos para los carcinomas de células claras ováricas (OCCC), dadas las opciones de tratamiento limitadas en esta enfermedad y la respuesta deficiente a la quimioterapia estándar. La sensibilidad de las células con deficiencia de ARID1A a dasatinib se asoció con el arresto del ciclo celular G1-S y dependía de p21 y Rb. Esto sugiere que dasatinib merece una investigación para el tratamiento de pacientes con CCC ARID1A-mutante. ${ }^{(12)}$

El carcinoma de células claras ováricos, como hemos plantado hasta ahora, se diferencia de otros tipos histológicos de cáncer de ovario epitelial, con alteraciones genéticas/epigenéticas, un perfil molecular específico relacionado con el sistema inmunitario y asociaciones epidemiológicas con etnia y endometriosis. Sin embargo, dos genes mutados principales son PIK3CA y ARID1A, que con frecuencia coexisten entre sí. Las alteraciones de otros genes también contribuyen a la activación del PI3K (por ejemplo, PIK3R1 y PTEN) y a la desregulación del complejo de remodelación de la cromatina (por ejemplo, ARID1B y SMARKA4). Aunque el número de variaciones del número de copia focal es pequeño en $\mathrm{CCCO}$, la amplificación se detecta recurrentemente en el cromosoma 20q13.2 (incluyendo ZNF217), 8q y 17q. Tanto el perfilado de expresión como el de metilación destacan la importancia de los ajustes al estrés oxidativo y a la inflamación. En particular, la regulación de los $\beta$ HNF-1 resultantes de la hipometilación contribuye al cambio del metabolismo anaeróbico a el metabolismo aeróbico de la glucosa. Además, la regulación de HNF-1 $\beta$ activa la señalización STAT3 y NF- $\kappa$, y conduce a la supresión inmune a través de la producción de IL-6 e IL-8. La supresión inmune también puede ser inducida por el aumento de la expresión de PD-1, Tim-3 y LAG3. Los tumores deficientes (microsatélites inestables) que se encuentran en el síndrome de Lynch también inducen la supresión inmunitaria en algunos casos. En un ensayo clínico reciente de fase II en cáncer de ovario resistente al platino fuertemente tratado, dos de cada veinte casos con una respuesta completa al anticuerpo anti-PD-1, nivolumab, fueron subtipos de CCC. Por lo tanto, el estado inmunosupresor resultante de las alteraciones genéticas y el microambiente tumoral único pueden estar asociados con la sensibilidad a los inhibidores del punto de control en esta entidad. ${ }^{(13)}$

Con respecto a la radioterapia Westhoff, et all. llevó a cabo un estudio en el que se tuvo en cuenta la naturaleza quimiorresistente de los tumores ginecológicos de células claras, investigando la utilidad de la radioterapia (RT) para tratar el carcinoma recurrente de células claras (CCC) del ovario. Para ello se llevó a cabo una revisión retrospectiva de los pacientes con CCC recurrente gestionados entre 1994 y 2012 en 2 centros médicos académicos. Como principales resultados de los 53 pacientes que tuvieron CCC 
recurrente, $24(45,3 \%)$ de estos pacientes recibieron RT. La supervivencia a los cinco años después de la recurrencia fue significativamente mayor en el grupo que recibió RT, $62,9 \%$ frente al 18,8\% que no la recibió (P a 0,002). Como conclusión del estudio la enfermedad sensible al platino y la RT se asocian con una mejor supervivencia. Sin embargo, es importante tener en cuenta que la mayoría de estos pacientes se sometieron a cirugía junto con RT, y puede ser que el beneficio de la RT se limita a aquellos que se someten a citorreducción secundaria. ${ }^{(14,15)}$

\section{Conclusiones}

- El carcinoma de células claras de ovario es un tipo histológico de cáncer de ovario epitelial cuya incidencia es muy baja.

- Las características clínicas son similares a los carcinomas de ovario más frecuentes a pesar de las diferencias en su perfil molecular.

- Frecuentemente resistente a la quimioterapia convencional por lo que su tratamiento requiere nuevos enfoques específicos.

\section{Referencias bibliográficas}

Kaku T, Ogawa S, Kawano Y, Ohishi Y, Kobayashi H, Hirakawa T, et al. Histological classification of ovarian cancer. Med electron Microsc Off J Clin Electron Microsc Soc Japan. marzo de 2003;36(1):9-17.

Lalwani N, Prasad SR, Vikram R, Shanbhogue AK, Huettner PC, Fasih N. Histologic, molecular, and cytogenetic features of ovarian cancers: implications for diagnosis and treatment. Radiogr a Rev Publ Radiol Soc North Am Inc. 2011;31(3):625-46.

Kobayashi H, Kajiwara H, Kanayama S, Yamada Y, Furukawa N, Noguchi T, et al. Molecular pathogenesis of endometriosis-associated clear cell carcinoma of the ovary (review). Oncol Rep. agosto de 2009;22(2):233-40.

del Carmen MG, Birrer M, Schorge JO. Clear cell carcinoma of the ovary: a review of the literature. Gynecol Oncol. septiembre de 2012;126(3):481-90.

Yamaguchi K, Mandai M, Oura T, Matsumura N, Hamanishi J, Baba T, et al. Identification of an ovarian clear cell carcinoma gene signature that reflects inherent disease biology and the carcinogenic processes. Oncogene. marzo de 2010;29(12):1741-52. 
Kato N, Sasou S, Motoyama T. Expression of hepatocyte nuclear factor-1beta (HNF1 beta) in clear cell tumors and endometriosis of the ovary. Mod Pathol an Off $\mathbf{J}$ United States Can Acad Pathol Inc. enero de 2006;19(1):83-9.

Iida Y, Okamoto A, Hollis RL, Gourley C, Herrington CS. Clear cell carcinoma of the ovary: a clinical and molecular perspective. Int $\mathbf{J}$ Gynecol cancer Off $\mathbf{J}$ Int Gynecol Cancer Soc. septiembre de 2020;

Lee Y-Y, Kim T-J, Kim M-J, Kim H-J, Song T, Kim MK, et al. Prognosis of ovarian clear cell carcinoma compared to other histological subtypes: a meta-analysis. Gynecol Oncol. septiembre de 2011;122(3):541-7.

Ku F-C, Wu R-C, Yang L-Y, Tang Y-H, Chang W-Y, Yang J-E, et al. Clear cell carcinomas of the ovary have poorer outcomes compared with serous carcinomas: Results from a single-center Taiwanese study. J Formos Med Assoc. febrero de 2018;117(2):117-25.

Kuroda T, Kohno T. Precision medicine for ovarian clear cell carcinoma based on gene alterations. Int J Clin Oncol. marzo de 2020;25(3):419-24.

Kuroda T, Ogiwara H, Sasaki M, Takahashi K, Yoshida H, Kiyokawa T, et al. Therapeutic preferability of gemcitabine for ARID1A-deficient ovarian clear cell carcinoma. Gynecol Oncol. diciembre de 2019;155(3):489-98.

Miller RE, Brough R, Bajrami I, Williamson CT, McDade S, Campbell J, et al. Synthetic Lethal Targeting of ARID1A-Mutant Ovarian Clear Cell Tumors with Dasatinib. Mol Cancer Ther. julio de 2016;15(7):1472-84.

Oda K, Hamanishi J, Matsuo K, Hasegawa K. Genomics to immunotherapy of ovarian clear cell carcinoma: Unique opportunities for management. Gynecol Oncol. noviembre de 2018;151(2):381-9.

Westhoff GL, Fuh KC, Longacre TA, McNally JL, Hsu I-C, Kapp DS, et al. Radiation Therapy for Recurrent Clear-Cell Cancer of the Ovary. Int J Gynecol cancer Off J Int Gynecol Cancer Soc. noviembre de 2016;26(9):1608-14.

Hsu YH, Wang PH, Chang CM. Functional gene clusters in global pathogenesis of clear cell carcinoma of the ovary discovered by integrated analysis of transcriptomes. Int J Environ Res Public Health. 2020 Jun 1;17(11).

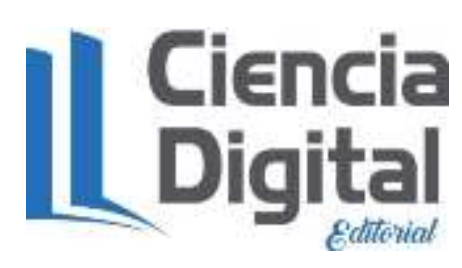


El artículo que se publica es de exclusiva responsabilidad de los autores y no necesariamente reflejan el pensamiento de la Revista Anatomía Digital.

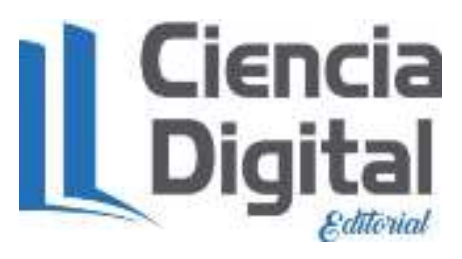

El artículo queda en propiedad de la revista y, por tanto, su publicación parcial y/o total en otro medio tiene que ser autorizado por el director de la Revista Anatomía Digital.
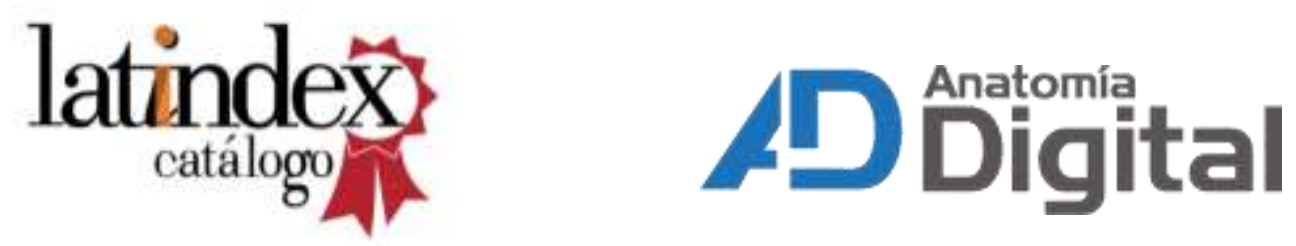

Indexacione:

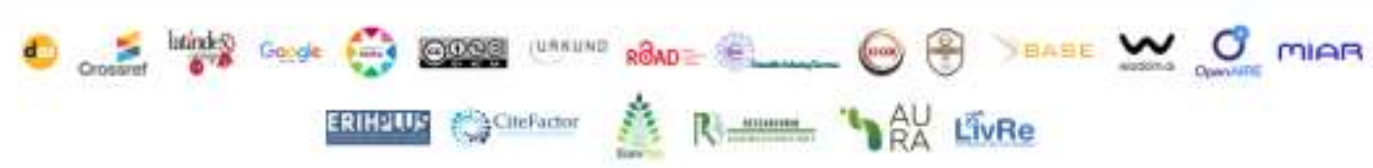

